\section{International Scientific Journal Theoretical \& Applied Science}

p-ISSN: $2308-4944$ (print)

e-ISSN: 2409-0085 (online)

Year: 2015

Issue: 04

Volume: 24

Published: 30.04.2015 http://T-Science.org
Alexandr Nikolayevich Shevtsov candidate of technical sciences, member of PILA (USA),

Department of «Mathematics», Taraz state University M.Kh. Dulati, Kazakhstan $\underline{\text { Shev_AlexXXXX@mail.ru }}$

SECTION 2. Applied mathematics. Mathematical modeling.

\title{
ABOUT THE BEHAVIOR OF THE TRAJECTORIES OF A DYNAMICAL SYSTEM WITH PURE IMAGINARY CHARACTERISTIC ROOTS NEAR THE STATE OF EQUILIBRIUM
}

Abstract: The paper discusses the dependence of trajectories of dynamical systems from the position of the center of the circle whose equation is in the right part of the system with purely imaginary characteristic roots.

Key words: dynamic system, center, trajectory.

Language: Russian

Citation: Shevtsov AN (2015) ABOUT THE BEHAVIOR OF ONE OF THE TRAJECTORIES OF A DYNAMICAL SYSTEM WITH PURE IMAGINARY CHARACTERISTIC ROOTS NEAR THE STATE OF EQUILIBRIUM. ISJ Theoretical \& Applied Science 04 (24): 272-280.

Soi: http://s-o-i.org/1.1/TAS*04(24)50 Doi: crossef http://dx.doi.org/10.15863/TAS.2015.04.24.50

\section{О ПОВЕДЕНИИ ТРАЕКТОРИЙ ОДНОЙ ДИНАМИЧЕСКОЙ СИСТЕМЫ С ЧИСТО МНИМЫМИ ХАРАКТЕРИСТИЧЕСКИМИ КОРНЯМИ ОКОЛО СОСТОЯНИЯ РАВНОВЕСИЯ}

Аннотация: В работе рассмотрены зависимости траекторий динамических систем от положения центра окружности, уравнение которой входит в правую часть системы с чисто мнимыми характеристическими корнями.

Ключевые слова: динамическая система, центр, траектория.

Рассмотрим динамическую систему, когда корни характеристического уравнения чисто мнимые [1, с.137].

$$
\frac{d x}{d t}=P(x, y), \quad \frac{d y}{d t}=Q(x, y) .
$$

Характеристическое уравнение системы (1) получим в виде (2).

$$
\left|\begin{array}{cc}
a-\lambda & b \\
c & d-\lambda
\end{array}\right|=\lambda^{2}-\sigma \lambda+\Delta=0
$$

Как известно [1-6] если $\lambda_{1}$ и $\lambda_{2}$ комплексные:

$$
\begin{aligned}
& \lambda_{1}=\alpha+i \beta, \\
& \lambda_{2}=\alpha+i \beta, \\
& \beta \neq 0,
\end{aligned}
$$

где $\alpha$ - может быть как равным, так и не равным нулю, то существует действительное неособое преобразование приводящее систему (1) к виду (4).

$$
\begin{aligned}
& \frac{d X}{d t}=\alpha X-\beta Y+\varphi_{1}(X, Y), \\
& \frac{d Y}{d t}=\beta X+\alpha Y+\psi_{1}(X, Y),
\end{aligned}
$$

Рассмотрим динамическую систему [6,8-9]:

$$
\begin{aligned}
& \frac{d x}{d t}=-y-x \sqrt{x^{2}+y^{2}}, \\
& \frac{d y}{d t}=x-y \sqrt{x^{2}+y^{2}} .
\end{aligned}
$$

где $\sqrt{x^{2}+y^{2}}=R$ - не что иное как окружность с радиусом $R$. Тогда (5) можно записать в виде:

$$
\begin{aligned}
& \frac{d x}{d t}=-y-x \cdot R, \\
& \frac{d y}{d t}=x-y \cdot R .
\end{aligned}
$$

Что по сути является системой (4), при следующих коэффициентах: 


$$
\begin{aligned}
& \alpha=0, \\
& \beta=1, \\
& \varphi_{1}(X, Y)=-x \cdot R, \\
& \psi_{1}(X, Y)=-y \cdot R .
\end{aligned}
$$

Систему будем решать в Maple, при начальных условиях (8) выводя три траектории (рис.1).

$$
\begin{aligned}
& t=0 . .150, \\
& \left.x\right|_{t=0}=0,\left.y\right|_{t=0}=1, \\
& \left.x\right|_{t=0}=1,\left.y\right|_{t=0}=1, \\
& \left.x\right|_{t=0}=1,\left.y\right|_{t=0}=\frac{1}{2} .
\end{aligned}
$$

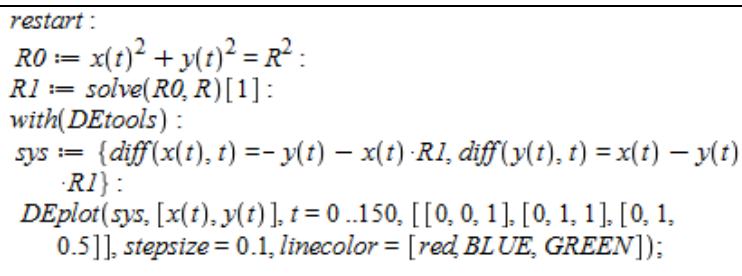

В случае $\sqrt{\left(x-x_{0}\right)^{2}+\left(y-y_{0}\right)^{2}}=R$ изменим программу следующим образом:

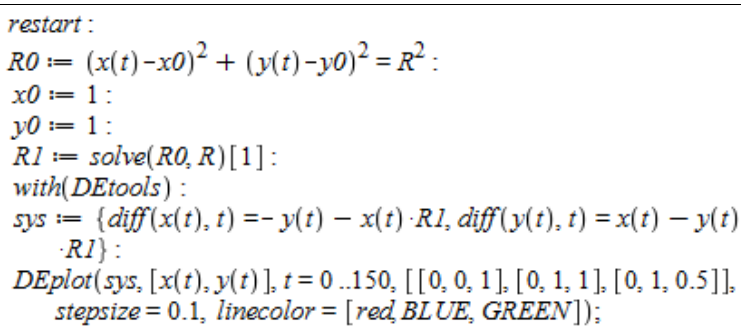

Исследуем поведение траекторий в зависимости от положения центра окружности на плоскости (рис. 2-4).

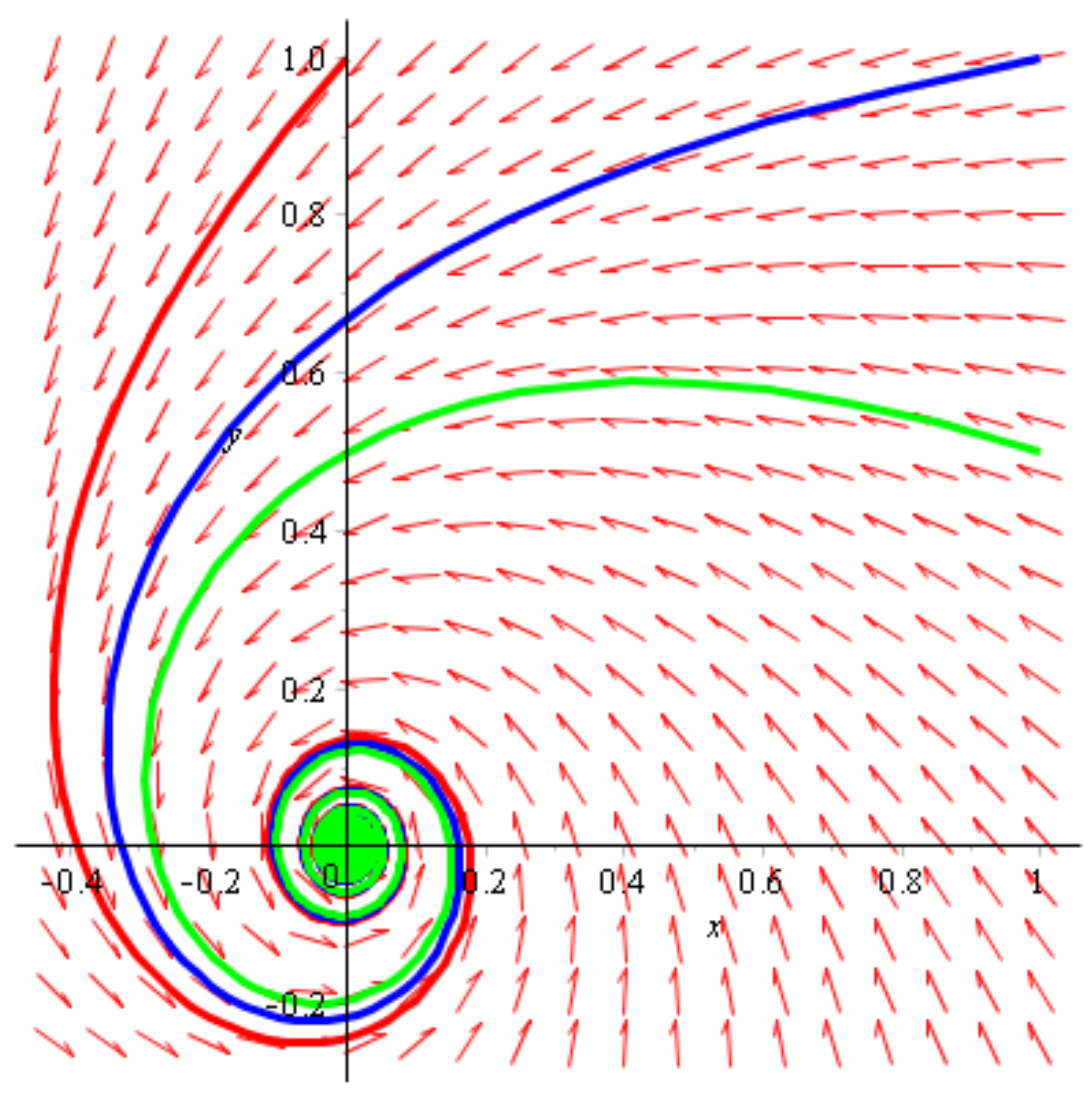

Рисунок 1 - Устойчивый фокус динамической системы. 
Impact Factor ISRA (India) $\quad=\mathbf{1 . 3 4 4}$

Impact Factor ISI (Dubai, UAE) $=\mathbf{0 . 8 2 9}$ based on International Citation Report (ICR)

Impact Factor GIF (Australia) $=\mathbf{0 . 3 5 6}$
Impact Factor JIF $\quad=\mathbf{1 . 5 0 0}$

Impact Factor SIS (USA) $\quad=\mathbf{0 . 9 1 2}$

Impact Factor РИНЦ (Russia) $=\mathbf{0 . 1 7 9}$

Impact Factor ESJI (KZ) $\quad=\mathbf{1 . 0 4 2}$

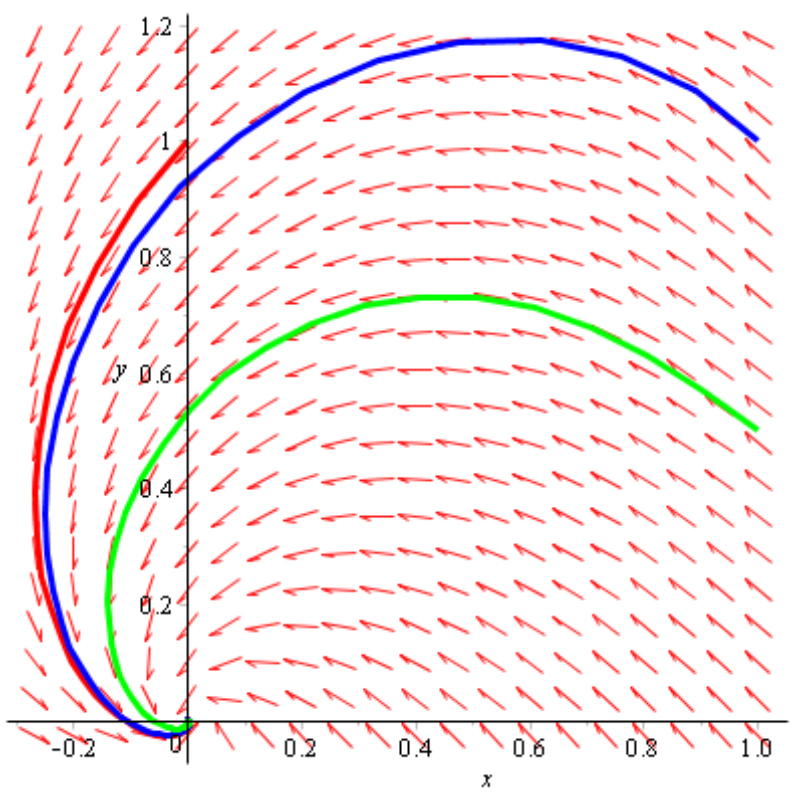

$(1,1)$

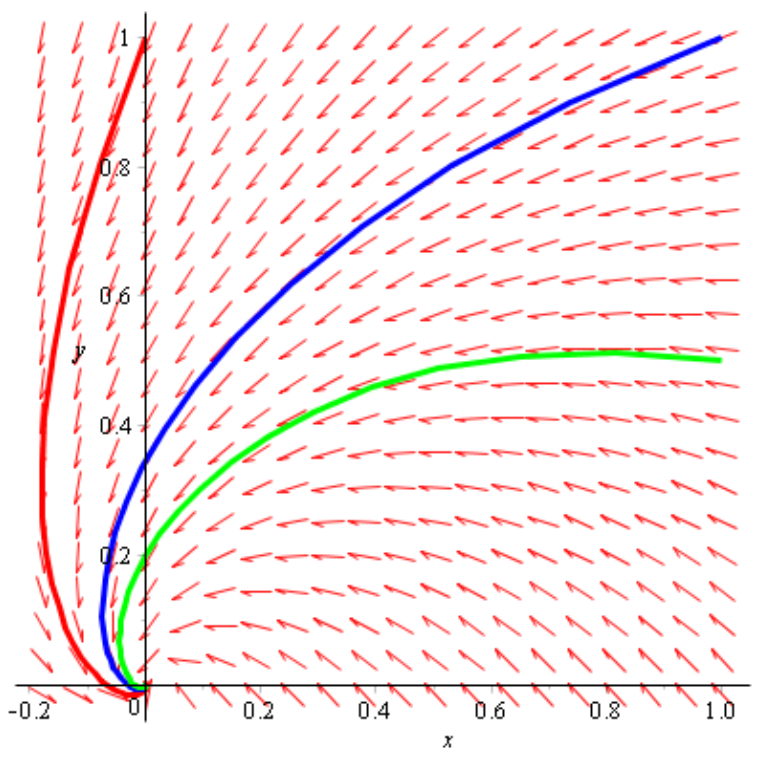

$(1,-1)$

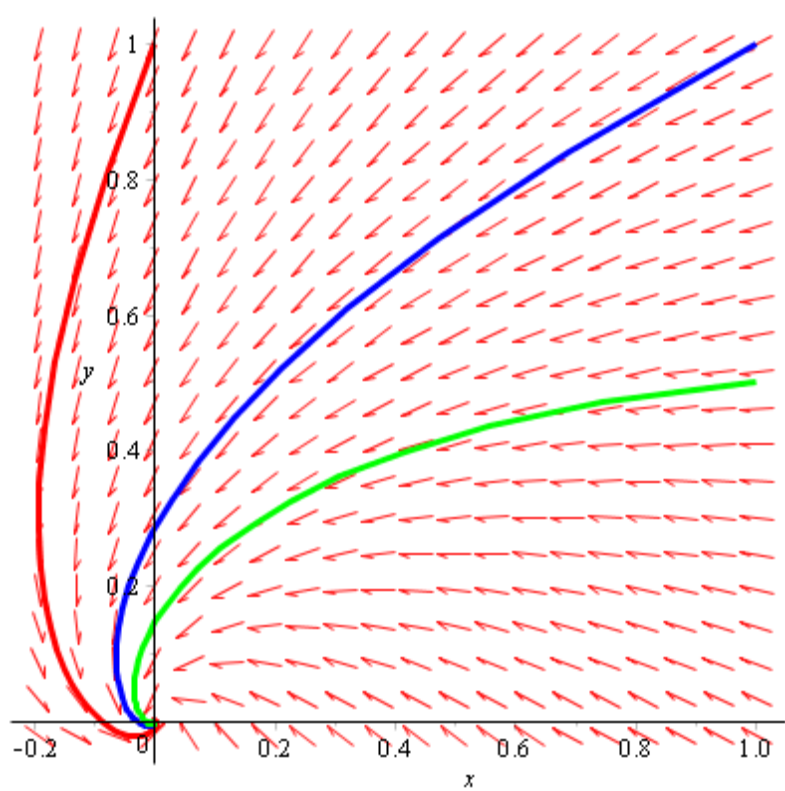

$(-1,-1)$

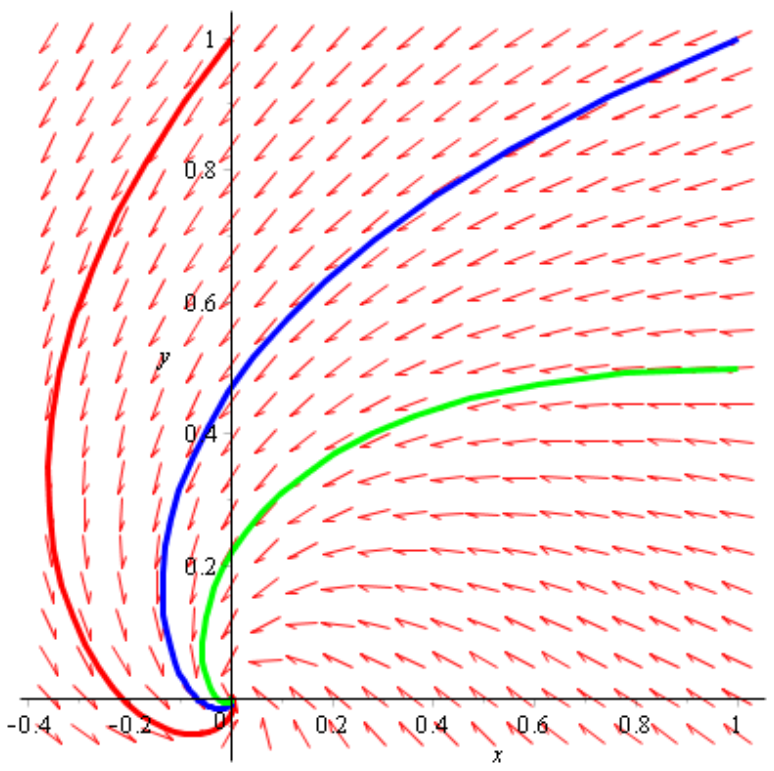

$(-1,1)$

Рисунок 2 - Зависимость векторного поля динамической системы от положения центра окружности. 
Impact Factor ISRA (India) $\quad=\mathbf{1 . 3 4 4}$

Impact Factor ISI (Dubai, UAE) $=\mathbf{0 . 8 2 9}$ based on International Citation Report (ICR)

Impact Factor GIF (Australia) $\quad \mathbf{0} \mathbf{0 . 3 5 6}$
Impact Factor JIF

$=\mathbf{1 . 5 0 0}$

Impact Factor SIS (USA) $\quad=\mathbf{0 . 9 1 2}$

Impact Factor РИНЦ (Russia) $=\mathbf{0 . 1 7 9}$

Impact Factor ESJI $(\mathrm{KZ}) \quad=\mathbf{1 . 0 4 2}$

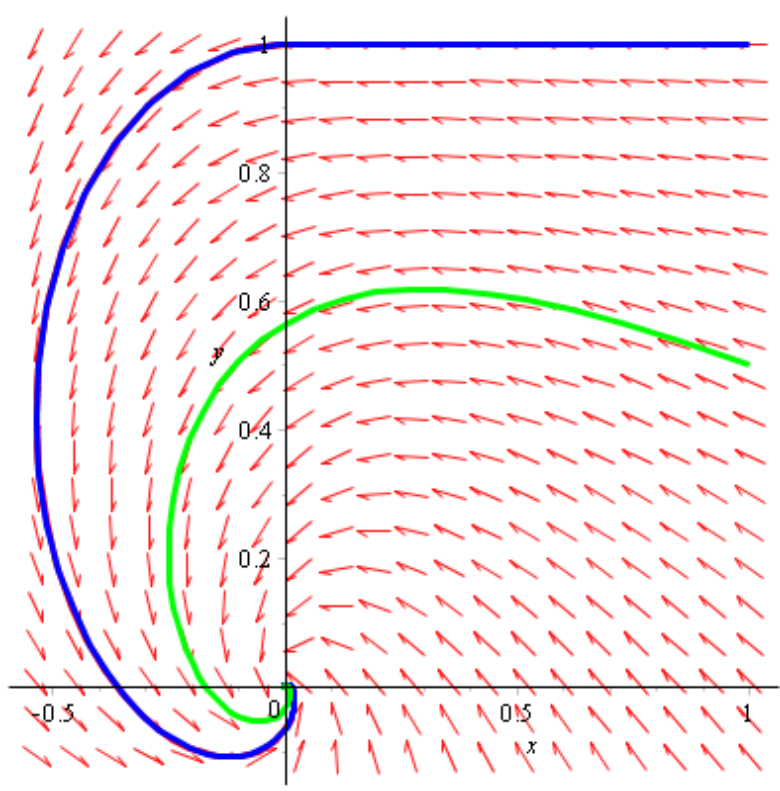

$(0,1)$

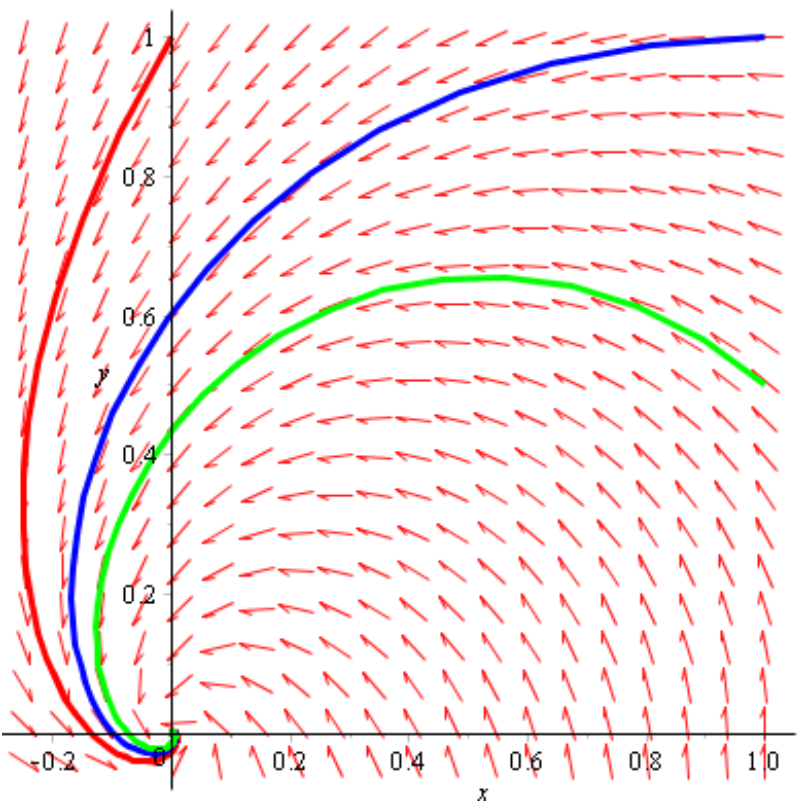

$(1,0)$

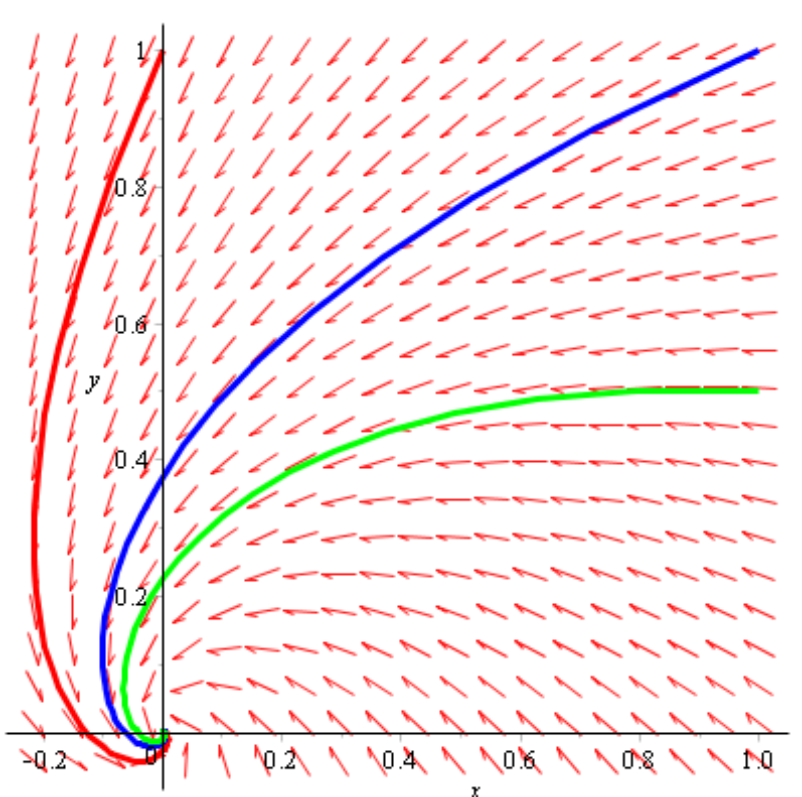

$(0,-1)$

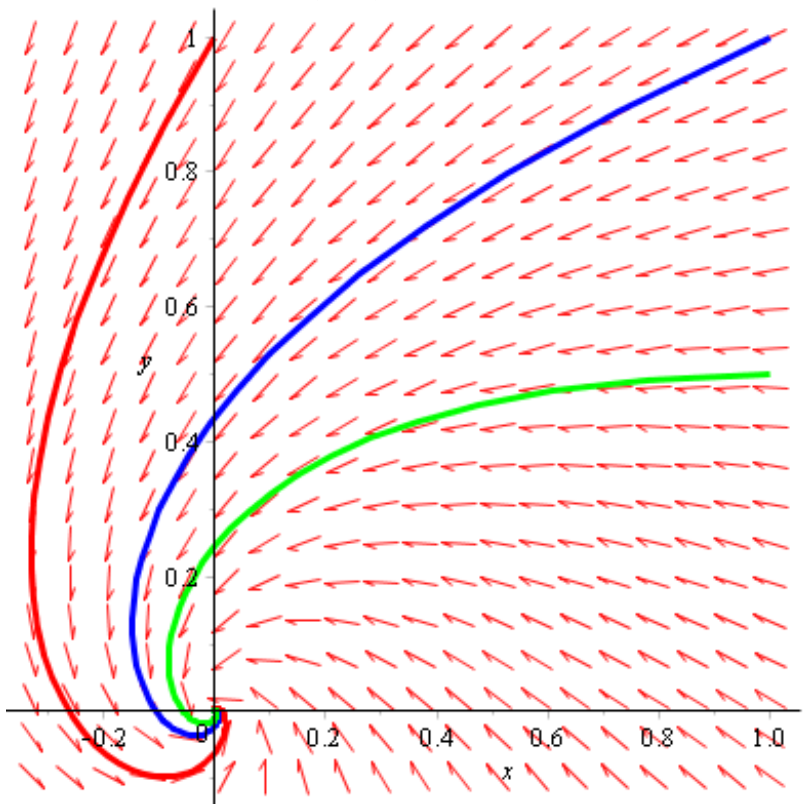

$(-1,0)$

Рисунок 3 - Зависимость векторного поля динамической системы от положения центра окружности.

ISPC The Combination of Technology \& Education, Östersund, Sweden 
Impact Factor ISRA (India) $\quad=\mathbf{1 . 3 4 4}$

Impact Factor ISI (Dubai, UAE) $=\mathbf{0 . 8 2 9}$ based on International Citation Report (ICR)

Impact Factor GIF (Australia) $=\mathbf{0 . 3 5 6}$
Impact Factor JIF

$=\mathbf{1 . 5 0 0}$

Impact Factor SIS (USA) $\quad=\mathbf{0 . 9 1 2}$

Impact Factor РИНЦ (Russia) = 0.179

Impact Factor ESJI (KZ) $\quad=\mathbf{1 . 0 4 2}$

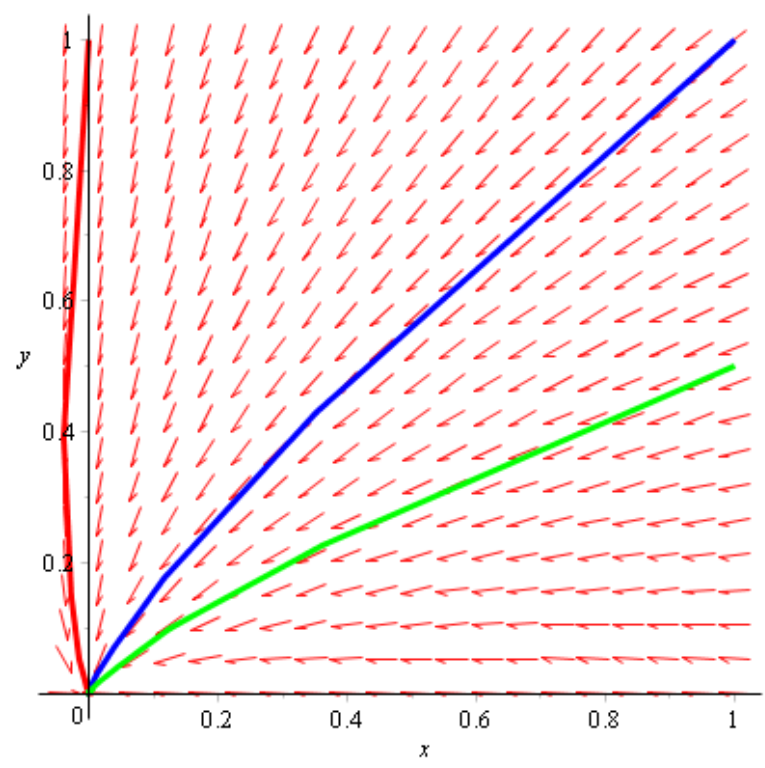

$(0,10)$

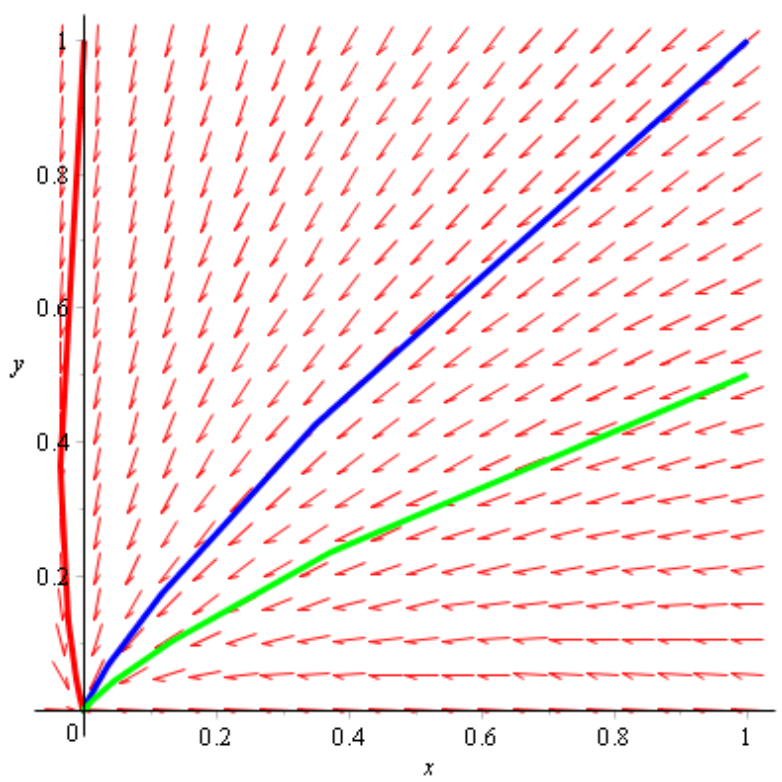

$(10,0)$

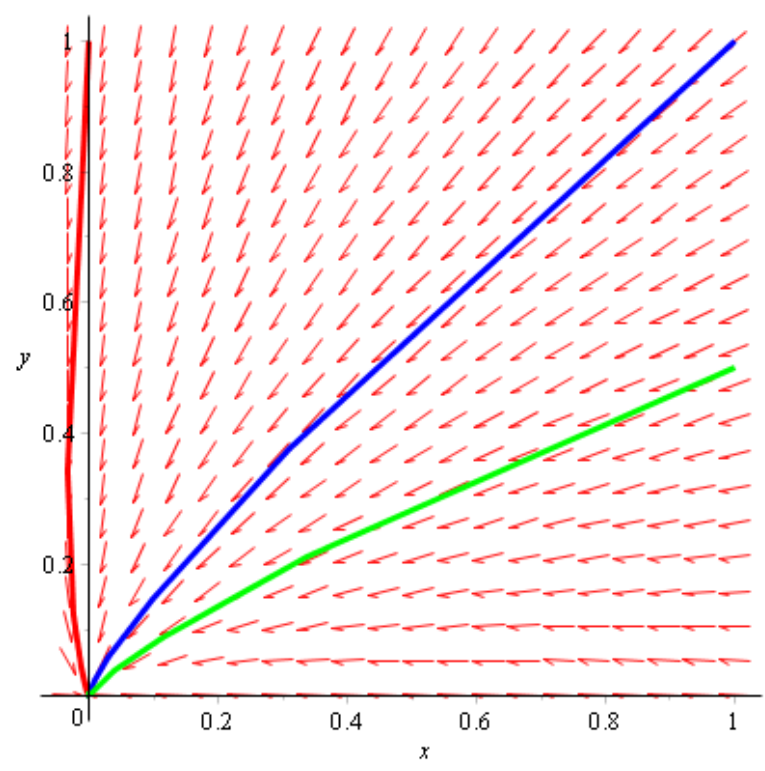

$(0,-10)$

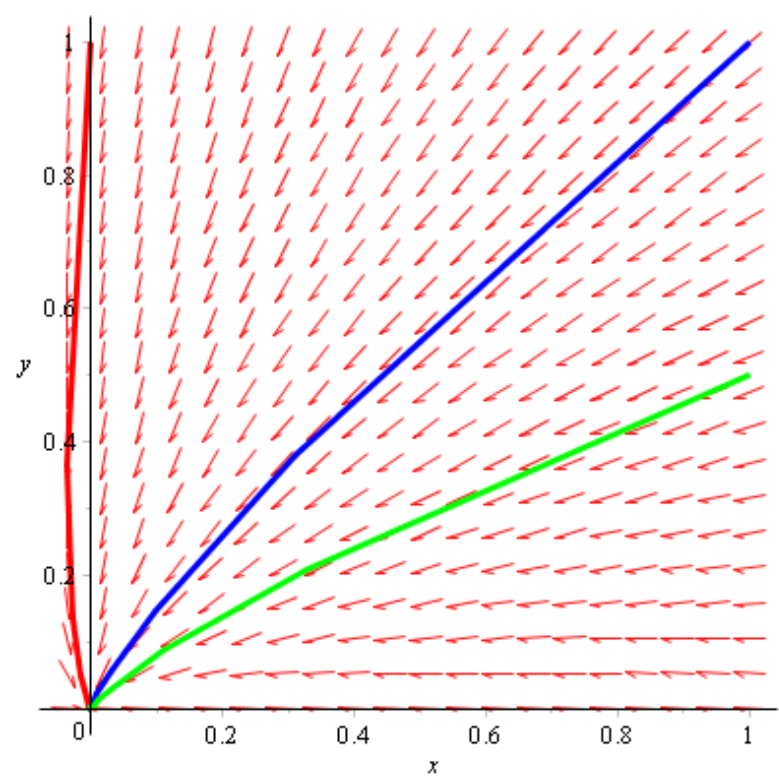

$(-10,0)$

Рисунок 4 - Смещения центра окружности на большие расстояния. 


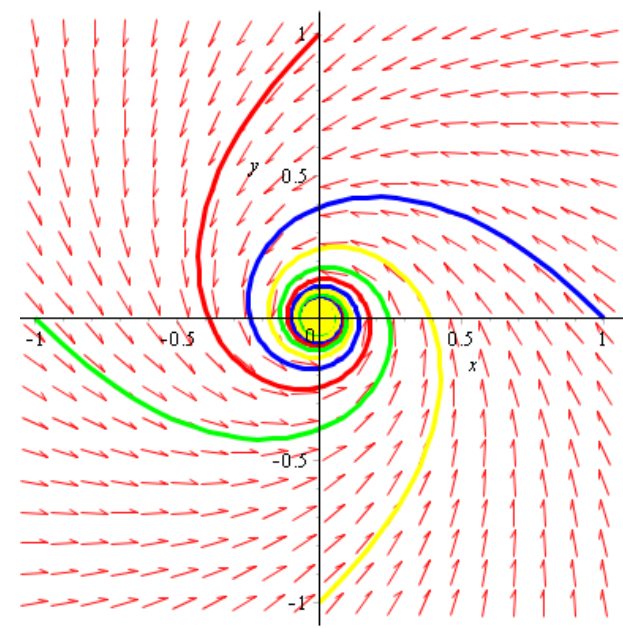

$(0,0)$

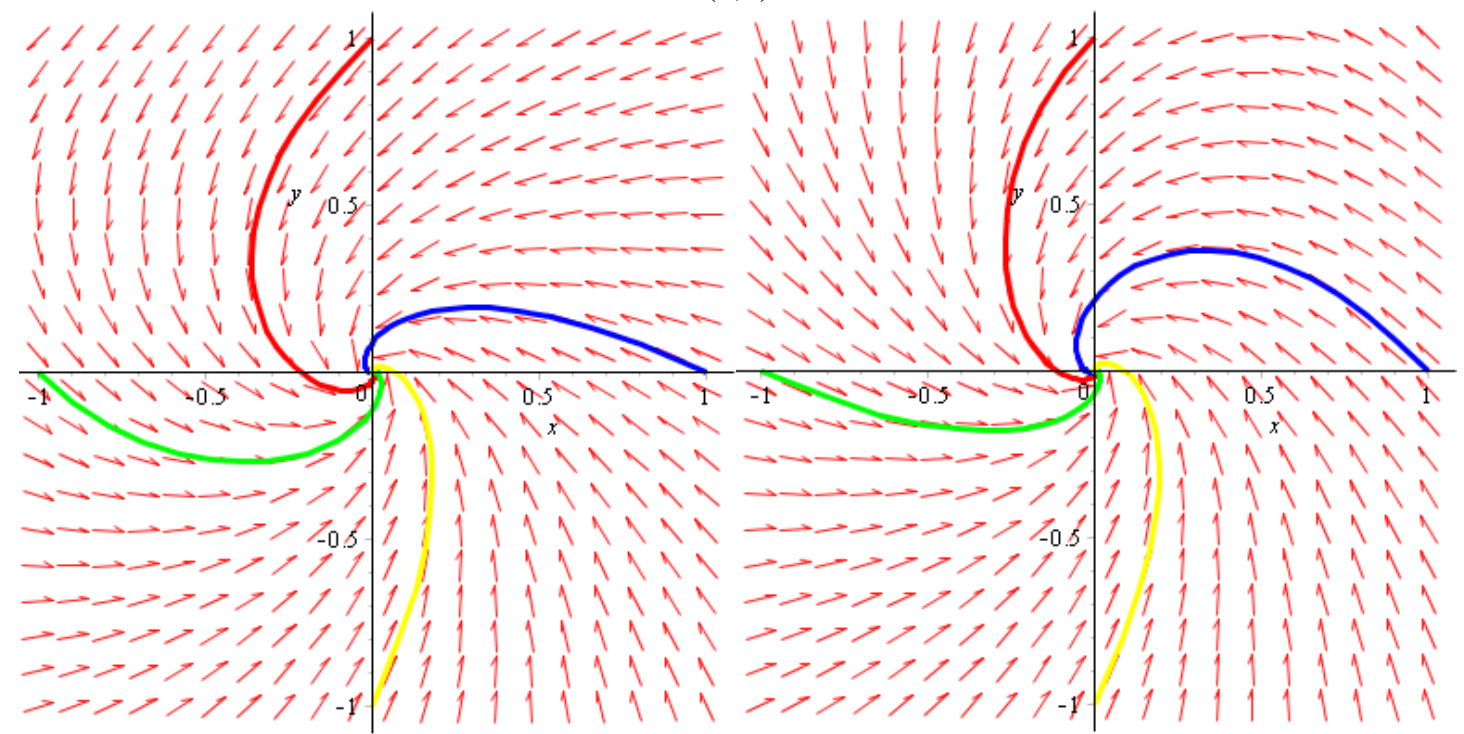

$(-1,1)$

$(1,1)$

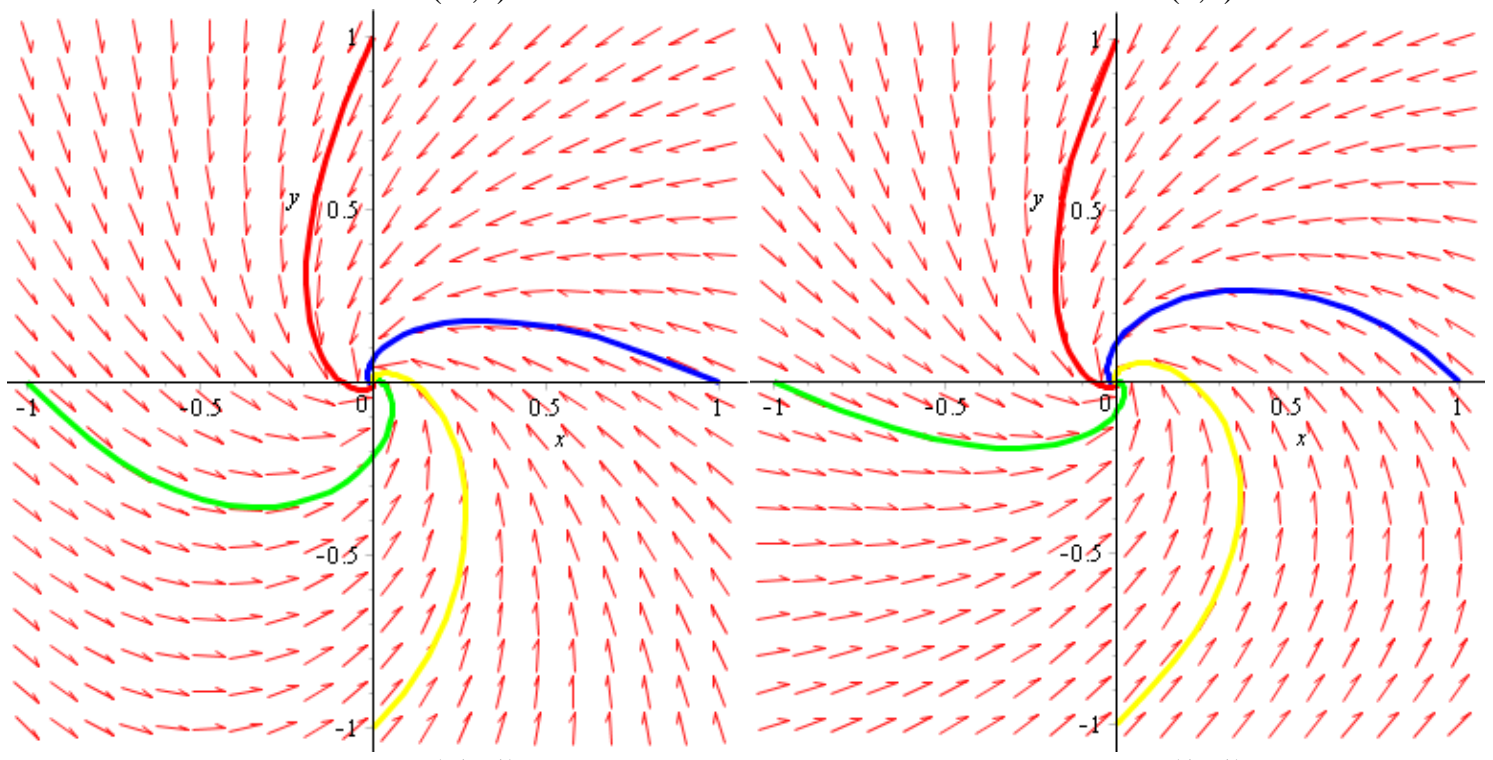

$(-1,-1)$

$(1,-1)$

Рисунок 5 - Сдвиг симметрии траекторий к центру окружности. 


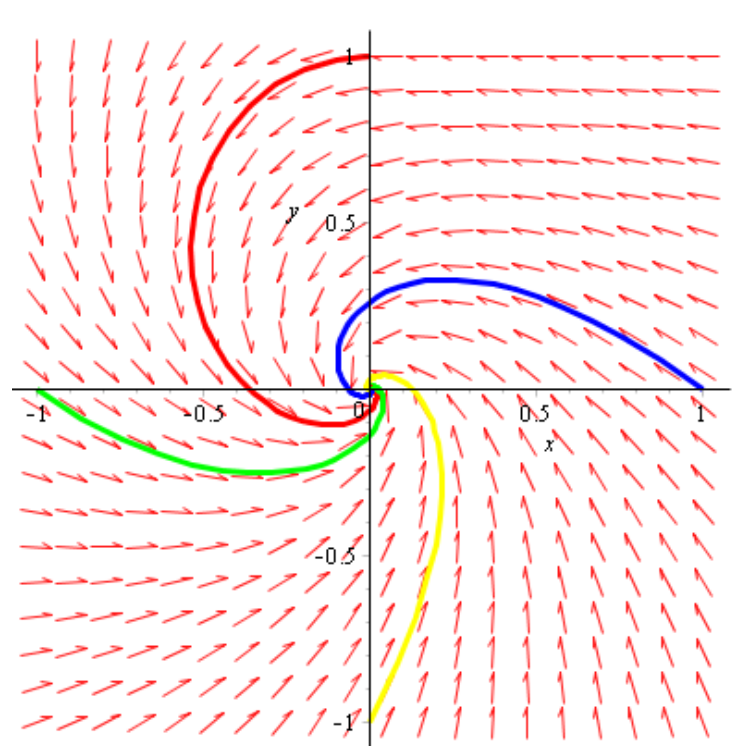

$(0,1)$

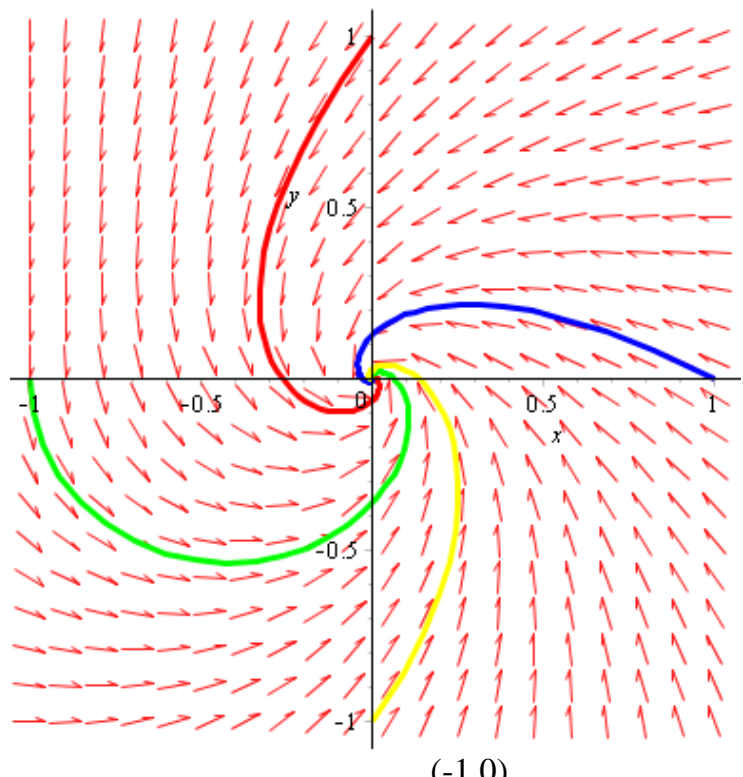

$(-1,0)$

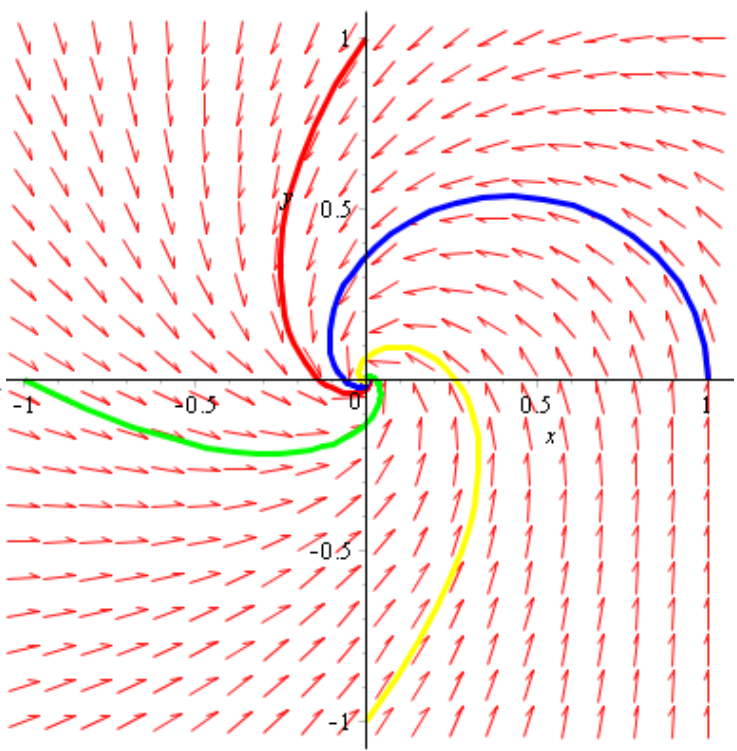

$(1,0)$

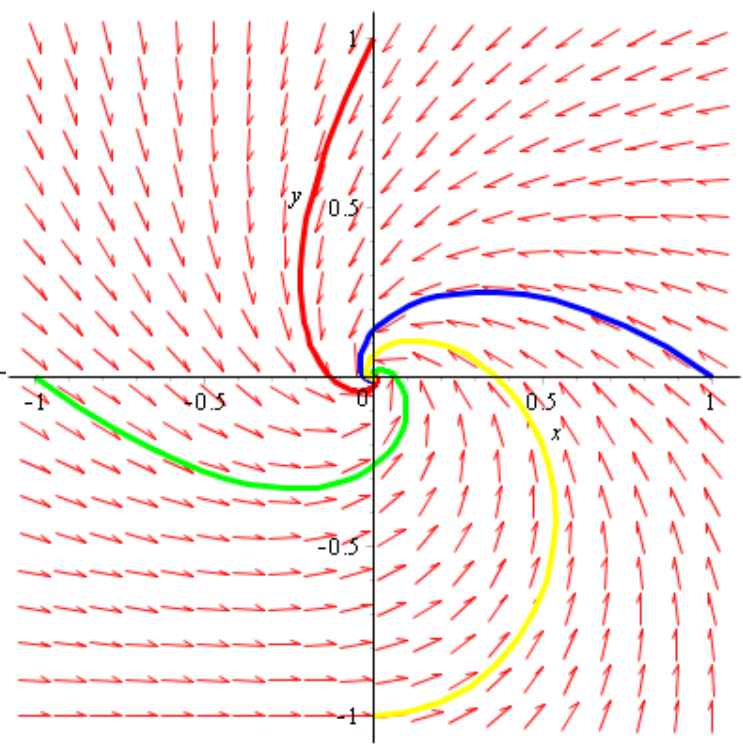

$(0,-1)$

Рисунок 6 - Сдвиг симметрии траекторий при совпадении центра окружности с началом траектории.

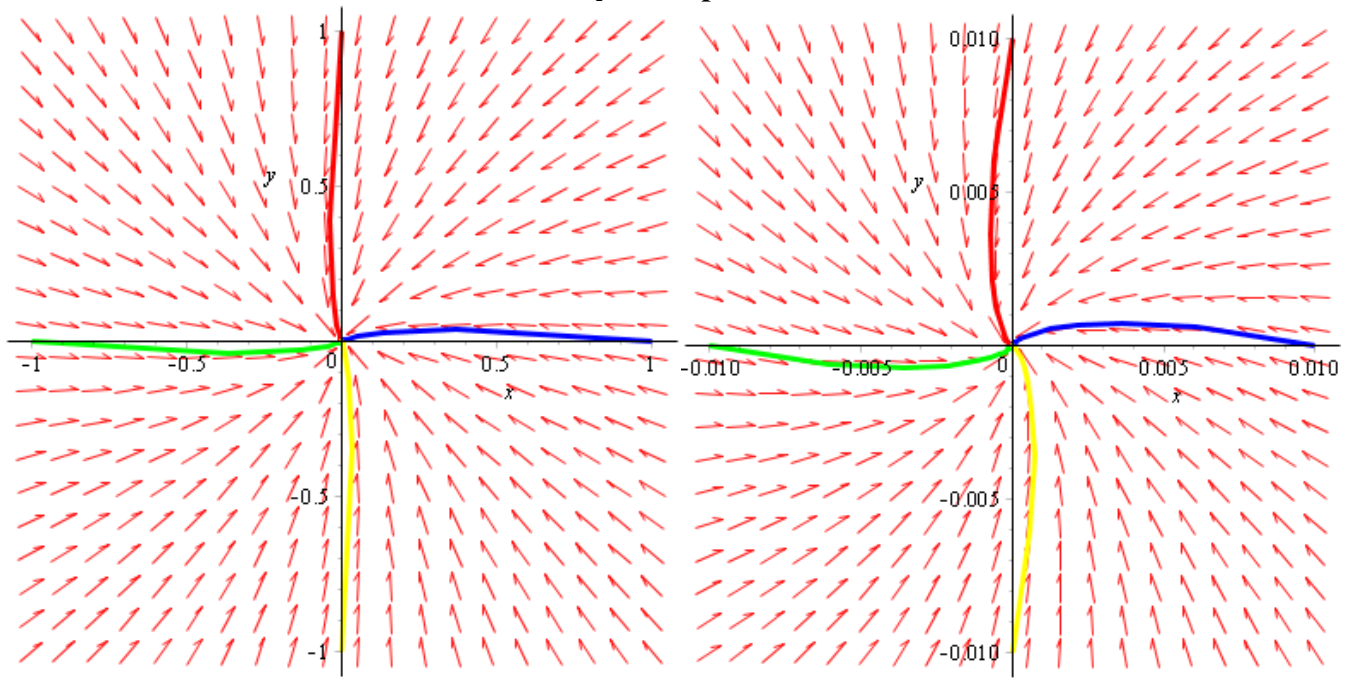

Рисунок 7 - Траектории не зависят от выбора масштаба расчета.

ISPC The Combination of Technology \&

Education, Östersund, Sweden 
Impact Factor ISRA (India) $\quad=\mathbf{1 . 3 4 4}$

Impact Factor ISI (Dubai, UAE) $=\mathbf{0 . 8 2 9}$

based on International Citation Report (ICR)

Impact Factor GIF (Australia) $\quad \mathbf{0} \mathbf{0 . 3 5 6}$
Impact Factor JIF

$=\mathbf{1 . 5 0 0}$

Impact Factor SIS (USA) $\quad=\mathbf{0 . 9 1 2}$

Impact Factor РИНЦ (Russia) $=\mathbf{0 . 1 7 9}$

Impact Factor ESJI $(\mathrm{KZ}) \quad=\mathbf{1 . 0 4 2}$
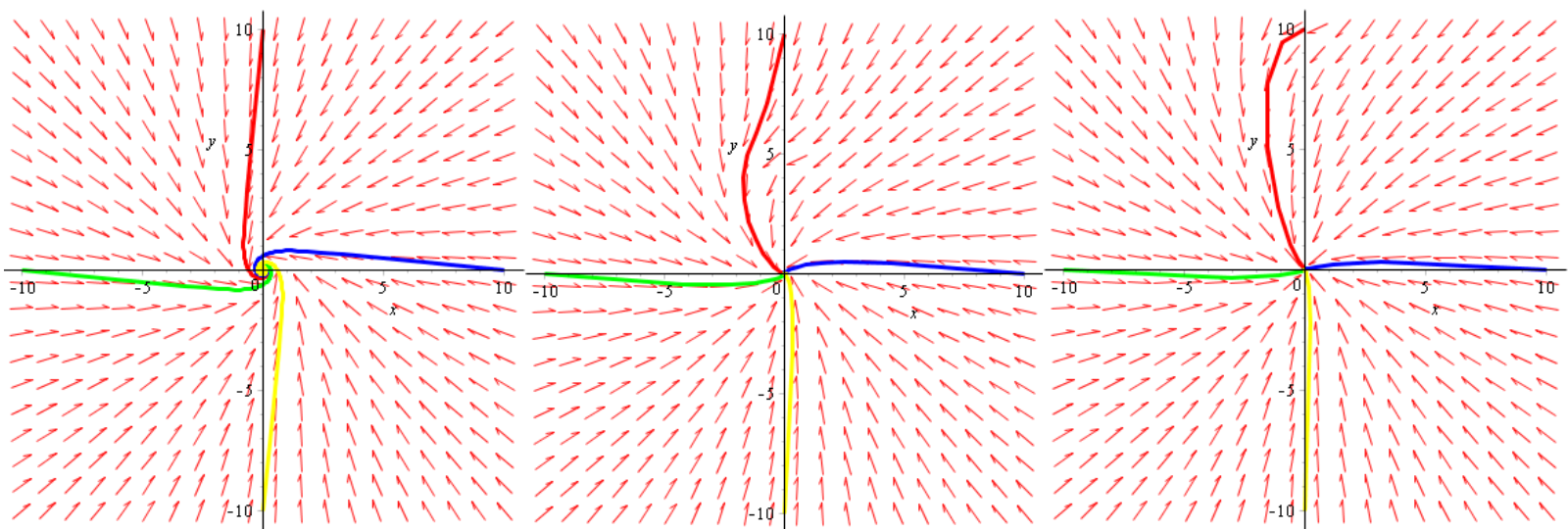

$(0,0)$

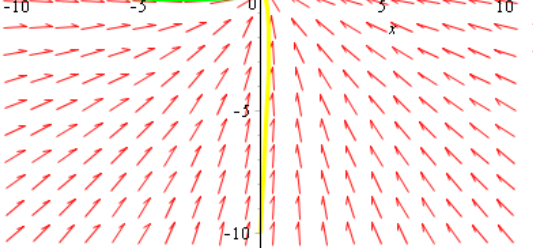

$(0,5)$

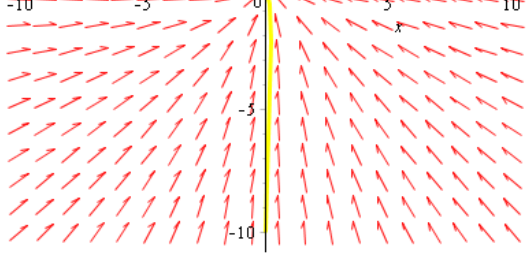

$(0,10)$

Рисунок 8 - Смещение центра вдоль оси Оу.

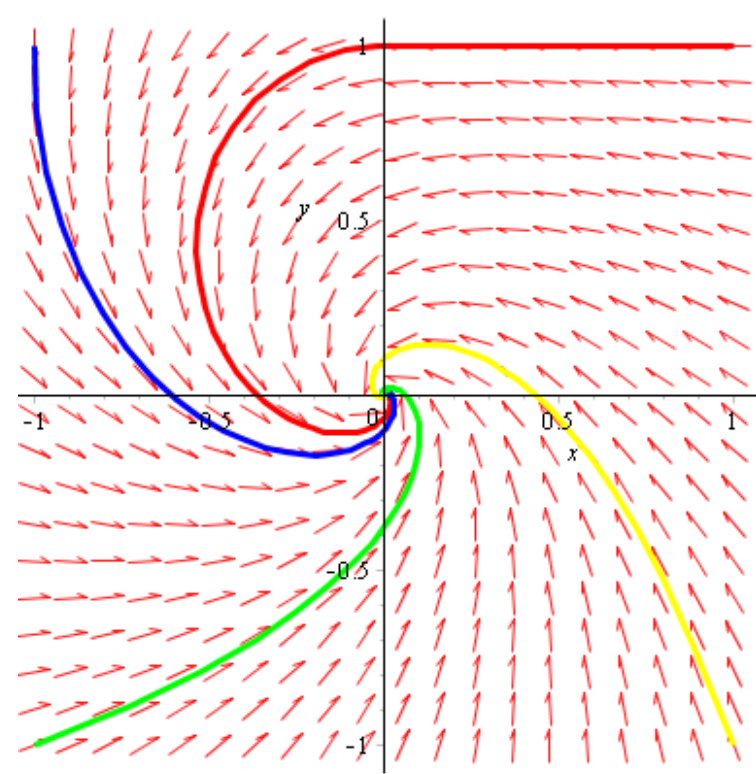

$(1,1)$

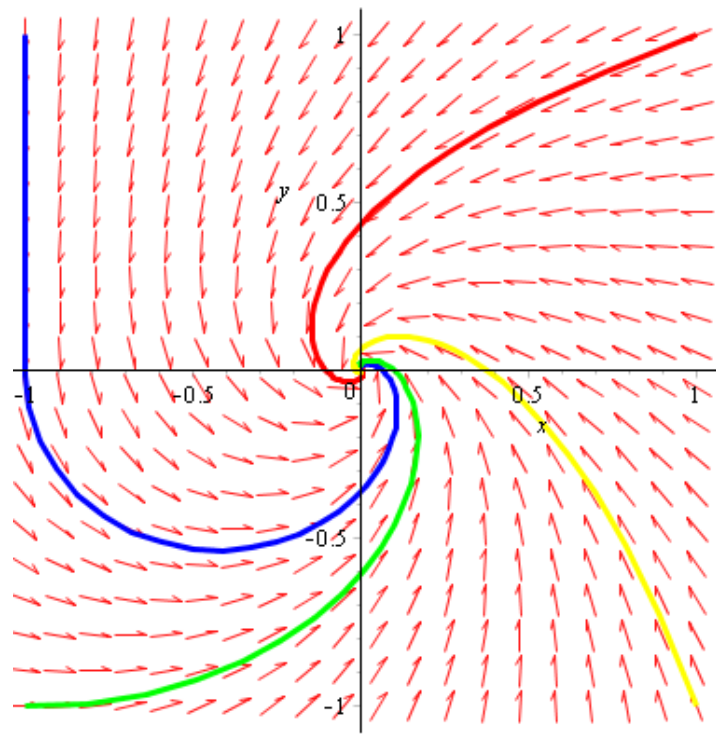

$(-1,1)$

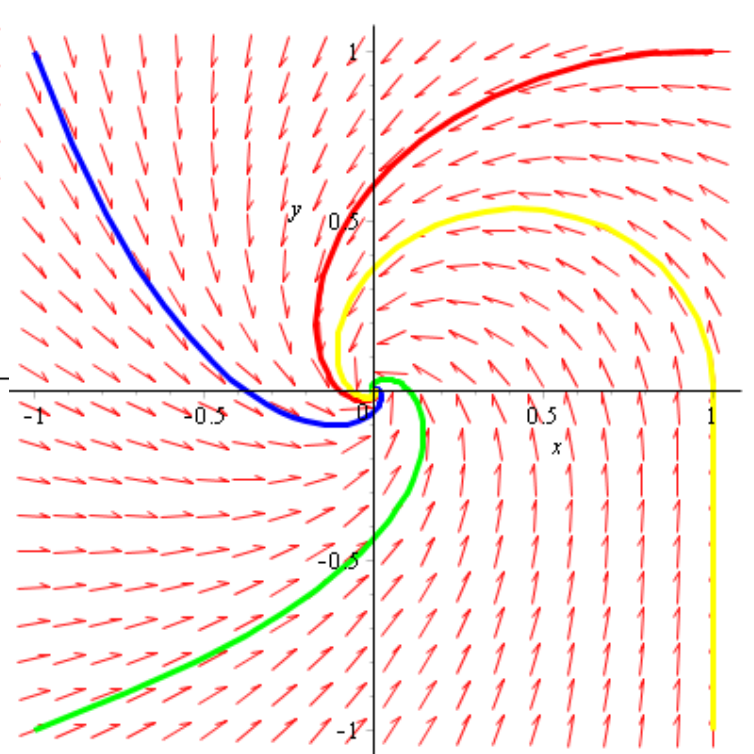

$(1,-1)$

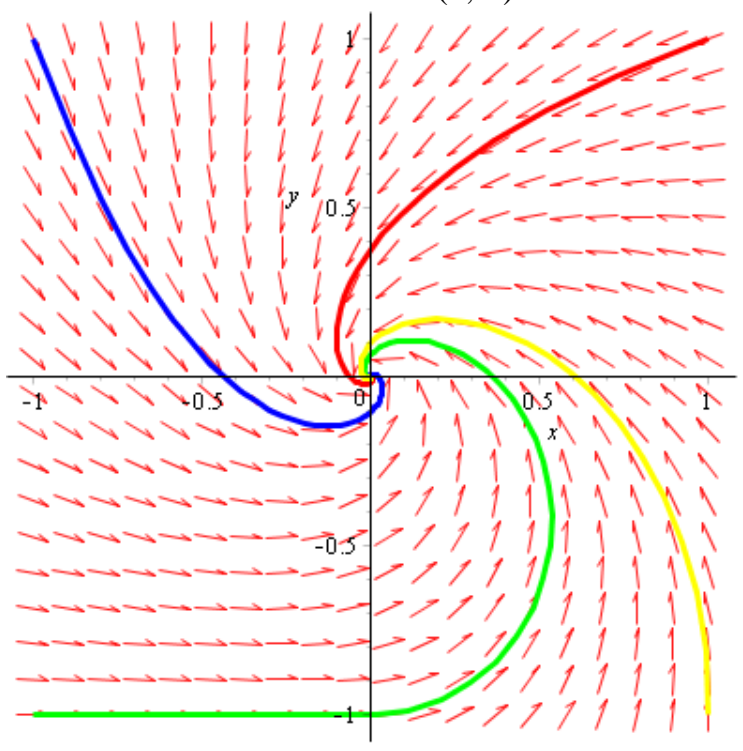

$(-1,-1)$

Рисунок 9 - Неизменность траекторий при выборе различных начальных точек.

ISPC The Combination of Technology \& Education, Östersund, Sweden 
Как видно из (рис.1-9) все траектории, вышедшие из разных начальных условий, асимптотически стремятся к одной и той же точке $(0,0)$. Но при этом (рис.5-6) траектории проходящие через центр окружности отклоняются намного сильнее.

Выбор масштаба для расчета (рис.7) не влияет на характер траекторий.

Смещение же центра окружности приводит к достаточно сильным отклонениям (рис.8).

Выбор начальных условий для траекторий не влияет ни на решение системы ни на характер
Impact Factor ESJI (KZ) $\quad=\mathbf{1 . 0 4 2}$

стремления к фокусу, меняя только сами траектории.

Полученные фазовые портреты дают исчерпывающую информацию для широкого диапазона начальных условий. Сходимость к другим аттракторам в ходе исследования не замечена.

Решение дифференциальной системы сходится к устойчивому фокусу при совпадении центра окружности с началом координат и к устойчивому узлу при отклонении.

\section{References:}

1. Andronov AA (1966) Qualitative theory of dynamical systems of the second order. Moscow: Nauka. -pp.58-68.

2. Bautin NN, Leontovich EA (1990) Methods and techniques of the qualitative study of dynamical systems on the plane. -Moscow. $484 \mathrm{p}$.

3. Malkin IG (1966) teorija ustoichivosti dvizhenija. - Moscow: Nauka. -533 p.

4. Kalitin BS (2002) Qualitative theory of stability of motion of dynamical systems. Minsk, -198 p.

5. Martynyuk AA (1990) Stability of motion. the method of limiting equations. Kiev, $-256 \mathrm{p}$.
6. Kosevich AM (1989) Keeping in nonlinear physical mechanics. Kiev, -304 p.

7. Kuznecov AP (2000) Kolebanija, katastrofy, bifurkacii, haos. Saratov, $-98 \mathrm{p}$.

8. Magnickij NA, Sidorov SV (2004) Novye metody haoticheskoj dinamiki. URSS, $-321 \mathrm{p}$.

9. Thompson JM (1982) Instabilities and Catastrophes in Science and Engineering. London, -254p.

10. Shapovalov AV (2002) Introduction To Nonlinear Physics. Tomsk, -129 p. 